INPLASY

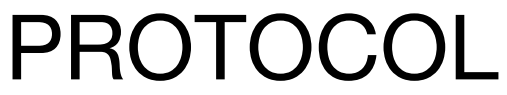

To cite: Wu et al. Effects and prognosis of photodynamic diagnosis in non-muscleinvasive bladder: a systematic review and meta-analysis. Inplasy protocol 2020110021. doi:

10.37766/inplasy2020.11.0021

Received: 05 November 2020

Published: 05 November 2020

Corresponding author:

Yukun Wu

wuyk27@mail.sysu.edu.cn

Author Affiliation:

The First Affiliated Hospital of

Sun Yat-sen University

Support: No.

Review Stage at time of this submission: The review has not yet started.

Conflicts of interest: None.

\section{Effects and prognosis of photodynamic diagnosis in non- muscle-invasive bladder: a systematic review and meta-analysis}

Wu, YK¹; Huang, B2 ; Chen, LW33.

Review question / Objective: Recent studies suggest that using the fluorescence photodynamic diagnosis (PDD) significantly improves diagnostic sensitivity with a positive influence upon the recurrence rate of NMIBC. The purpose of this research is to systematically review the effects and prognosis of PDD on bladder cancer clinical outcomes.

Condition being studied: Previous studies showed the use of PDD for patients with NMIBC results in a significant $26 \%$ diagnostic sensitivity improvement as well as superior patient prognosis and quality of life following conservative treatment by reducing the tumor recurrence rate with up to $20 \%$ after five years of follow-up (Petru et al., 2017). However, how patients benefited from PDD still had some arguments.

INPLASY registration number: This protocol was registered with the International Platform of Registered Systematic Review and Meta-Analysis Protocols (INPLASY) on 05 November 2020 and was last updated on 05 November 2020 (registration number INPLASY2020110021).

\section{INTRODUCTION}

Review question / Objective: Recent studies suggest that using the fluorescence photodynamic diagnosis (PDD) significantly improves diagnostic sensitivity with a positive influence upon the recurrence rate of NMIBC. The purpose of this research is to systematically review the effects and prognosis of PDD on bladder cancer clinical outcomes.

Condition being studied: Previous studies showed the use of PDD for patients with 
NMIBC results in a significant $26 \%$ diagnostic sensitivity improvement as well as superior patient prognosis and quality of life following conservative treatment by reducing the tumor recurrence rate with up to $20 \%$ after five years of follow-up (Petru et al., 2017). However, how patients benefited from PDD still had some arguments.

\section{METHODS}

Search strategy: The following databases will be searched: PubMed, EMBASE, Web of Science, Cochrane Library, CBM, CNKI, and Wanfang databases. We have developed a search strategy that combines keywords with medical subject words (MESH) / EMTREE terms. The following expressions will be used: (bladder cancer or bladder carcinoma *) and (PDD or Hexvix or fluorescein * or 5-ALA or HAL or Photodyn*) and (randomized controlled trials or clinical trials). The search is limited to human research, and there are no language restrictions.

Participant or population: Patients who had been diagnosed as NMIBC.

Intervention: Photodynamic diagnosis.

Comparator: White light cystoscopy.

Study designs to be included: RCTs.

Eligibility criteria: The development and implementation of the research program will be in line with the preferred reporting project for system review and metaanalysis reports. The study included in our meta-analysis will meet all the following inclusion criteria in the PICOS ranking: (1) patients suspected or diagnosed with NMIBC; (2) intervention: photodynamic diagnosis group; (3) control intervention: White light cystoscopy group; (4) outcome indicators: at least one of the following outcome indicators should be reported: recurrence, progression, recurrence-free survival or (CIS) detection rate of cancer in situ. (5) study design: randomized controlled trial (RCT). Articles that do not evaluate the above results or do not compare the two groups will not be included in this meta-analysis. Duplicate reports and meeting summaries will be excluded. Retrospective trials, case reports, biochemical tests, letters and reviews will also be cancelled. Two independent authors will screen titles and abstracts of potentially relevant studies to determine their qualifications according to the criteria. The differences will be resolved through discussions with third review author.

Information sources: The following databases will be searched: PubMed, EMBASE, Web of Science, Cochrane Library, CBM, CNKI, and Wanfang databases.

Main outcome(s): Recurrence rate of bladder cancer, complication of the surgery.

Additional outcome(s): Progression rate, recurrence-free survival, and carcinoma in situ (CIS) detection rate.

Quality assessment / Risk of bias analysis: Two reviewers independently assessed the quality of the included studies using a version of the QUOROM guidelines.

Strategy of data synthesis: According to the basic characteristics of the included study, Review Manager 5.3 will be used for Meta-analysis. Considering the characteristics of the data extracted from the review, the continuous results will be expressed as the average of $95 \%$ confidence interval (Cl). Differences in classification variables are expressed as risk ratio values and 95 per cent $\mathrm{Cl}$. Heterogeneity will be assessed by 12 statistics. $12 \geq 50 \%$ represents a high degree of heterogeneity.

Subgroup analysis: We will conduct a subgroup analysis based on the use of photosensitizer (5-ALA or HLA) and the grade of bladder cancer. 
Sensibility analysis: Sensitivity analyses will be undertaken to determine the potential source of heterogeneity when significant.

Country(ies) involved: China.

Keywords: bladder cancer, photodynamic diagnosis, 5-aminolevulinic acid, hexylaminolevulinate.

Contributions of each author:

Author 1 - Yukun Wu.

Author 2 - Bin Huang.

Author 3 - Lingwu Chen. 